\title{
COMMUNICATIONS
}

\section{Scaling of the memory function and Brownian motion}

\author{
G. R. Kneller \\ Centre de Biophysique Moléculaire, CNRS UPR 4301 Rue Charles Sadron, \\ F-45071 Orléans Cedex 2, France
}

G. Sutmann

Central Institute for Applied Mathematics (ZAM) and John von Neumann Institute for Computing (NIC) Research Centre Jülich, D-52425 Jülich, Germany

(Received 17 October 2003; accepted 26 November 2003)

\begin{abstract}
It has been recently shown that the velocity autocorrelation function of a tracer particle immersed in a simple liquid scales approximately with the inverse of its mass [J. Chem. Phys. 118, 5283 (2003)]. With increasing mass the amplitude is systematically reduced and the velocity autocorrelation function tends to a slowly decaying exponential, which is characteristic for Brownian motion. We give here an analytical proof for this behavior and comment on the usual explanation for Brownian dynamics which is based on the assumption that the memory function is proportional to a Dirac distribution. We also derive conditions for Brownian dynamics of a tracer particle which are entirely based on properties of its memory function. (C) 2004 American Institute of Physics. [DOI: 10.1063/1.1642599]
\end{abstract}

Recently, a numerical method was published which allows one to extract memory functions reliably from molecular dynamics simulations. ${ }^{1,2}$ The concept of memory functions was introduced by Zwanzig to describe the dynamics of liquids in terms of a generalized Langevin equation., ${ }^{3,4}$ The method proposed in Refs. 1 and 2 is based on an autoregressive model for the time series of the dynamical variable under consideration. This approach was used to examine under which conditions the dynamics of a tracer particle in a simple solvent can be described by Brownian dynamics. ${ }^{5}$ For this purpose both the size and the mass of the tracer particle were varied independently. It was demonstrated that the size of a tracer particle strongly influences the form of its velocity memory function, whereas a change of its mass leads essentially to a global scaling of the latter. With increasing mass the amplitude of the memory function is reduced and the corresponding velocity autocorrelation function (VACF) tends to a slowly decaying exponential. The smaller the tracer particle, the stronger the effect. The scaling behavior for the memory function is exactly understood for time $t$ $=0,{ }^{4}$ and more subtle finite size effects predicted by Español and Zuñiga ${ }^{6}$ could be reproduced in Ref. 5. As far as we know, a scaling of the memory function for all times upon a change of the particle mass has not yet been reported.

Usually the tendency towards an exponential VACF is explained by assuming an increasingly short-ranged memory function, whereas we find that the amplitude of the memory function is globally reduced with increasing mass. This is not a contradiction, since the memory function decays rapidly relative to the VACF. To discuss this point in more detail we start from the memory function equation for the VACF which has been derived by Zwanzig ${ }^{3,4}$

$$
\dot{\psi}(t)=-\int_{0}^{t} d \tau \xi(t-\tau) \psi(\tau) .
$$

Here $\psi(t)$ is the normalized VACF of one Cartesian component $v(t)$ of the particle velocity

$$
\psi(t)=\frac{\langle v(t) v(0)\rangle}{\left\langle v^{2}\right\rangle}
$$

and $\xi(t)$ is the corresponding memory function. The latter can be expressed as $\xi(t)=\langle\dot{v} \exp (i[1-\mathcal{P}] \mathcal{L} t) \dot{v}\rangle /\left\langle v^{2}\right\rangle$. Here $\mathcal{L}$ is the Liouville operator of the system and $\mathcal{P}$ is a projector. Its action on an arbitrary function in phase space $f$ is defined through $\mathcal{P} f=v\langle v f\rangle /\left\langle v^{2}\right\rangle$. Details about the derivation of $\xi(t)$ can be found in the book of Boon and Yip. ${ }^{4}$ It is important to note that the memory function equation defined in Eq. (1) is exact. The classical Brownian dynamics approximation of $\psi(t)$ is retrieved by setting $\xi(t)=\gamma \delta(t)$, such that

$$
\dot{\psi}(t)=-\gamma \psi(t)
$$

and $\psi(t)=\exp (-\gamma t)$. It must be emphasized that an exponential form for $\psi(t)$ is an approximation. Since $\psi(t)$ is an even differentiable function it follows that

$$
\dot{\psi}(0)=0 .
$$

With $\psi(t)=\exp (-\gamma t)$ one finds, however, $\dot{\psi}(0)=-\gamma$. This contradiction shows that an exponentially decaying VACF cannot be valid on all time scales and motivates the introduction of a coarse grained time scale for Brownian dynamics.

Let us now study the influence of a global scaling of the memory function, as observed in our computer simulations, and the effect on the corresponding VACF. We emphasize that we do not aim at giving an explanation for the scaling behavior of $\xi(t)$. The goal is here to show why the observed scaling behavior leads to exponential VACFs. For this purpose we consider the Laplace transform of $\psi(t)$, which is defined as 


$$
\hat{\psi}(s)=\int_{0}^{\infty} d t \exp (-s t) \psi(t), \quad \mathfrak{R}\{s\}>0 .
$$

Because of Eq. (1) one has

$$
\hat{\psi}(s)=\frac{1}{s+\hat{\xi}(s)} .
$$

If we change $\xi(t) \rightarrow \alpha \xi(t)$, where $\alpha>0$, it follows from the linearity of the Laplace transform that $\hat{\xi}(s) \rightarrow \alpha \hat{\xi}(s)$, and therefore

$$
\begin{aligned}
\psi_{\alpha}(t) & =\frac{1}{2 \pi i} \oint_{C} d s \frac{\exp (s t)}{s+\alpha \hat{\xi}(s)}, \\
& =\frac{1}{2 \pi i} \oint_{C^{\prime}} d s \frac{\exp (s \alpha t)}{s+\hat{\xi}(\alpha s)} .
\end{aligned}
$$

The last line shows that $\psi_{\alpha}(t)$ is obtained in two steps:

(i) The memory function is rescaled as

$$
\xi(t) \rightarrow \frac{1}{\alpha} \xi\left(\frac{t}{\alpha}\right) .
$$

Here one uses the scaling property of the Laplace transform $(1 / \alpha) f(t / \alpha) \leftrightarrow \hat{f}(\alpha s)(\alpha>0)$, for any function $f(t)$ whose Laplace transform $\hat{f}(s)$ exists.

(ii) The rescaled memory function in Eq. (7) is used to evaluate the corresponding VACF on the "stretched" time scale $t \rightarrow \alpha t$.

If the integral of $\xi(t)$ exists, such that

$$
\int_{0}^{\infty} d t \xi(t) \equiv \gamma<\infty,
$$

the scaling applied in Eq. (7) leads to $\xi(t) \approx \gamma \delta(t)$ if $\alpha \ll 1$. Since the corresponding VACF is evaluated on the time scale $t \rightarrow \alpha t$, one obtains

$$
\psi_{\alpha}(t) \approx \exp (-\alpha \gamma t) .
$$

The limit $\alpha \rightarrow 0$ is not meaningful since the corresponding exact VACF would not decay at all. This is in agreement with property (4) of $\psi(t)$ and simply indicates that Brownian motion must be described on a coarse grained time scale $t$ $=n \Delta t(n=0,1,2, \ldots)$. The exponential decay of the VACF must be considered on that time scale.

We will now discuss criteria that allow us to determine $\Delta t$ and to predict the validity of the exponential model from the memory function. In a discrete model an exponentially decaying VACF is the solution of the difference equation

$$
\frac{\psi(t+\Delta t)-\psi(t)}{\Delta t}=-\gamma \psi(t),
$$

which replaces the differential equation (3). If we set

$$
a=1-\gamma \Delta t,
$$

the solution of the difference equation (10) takes the simple form

$$
\psi(n \Delta t)=a^{n} .
$$

If $\gamma \Delta t<1$, the VACF has the form of a discrete decaying exponential, and for $\gamma \Delta t \ll 1$ one approximates a continuous exponential function.

A VACF which has the form given in Eq. (12) is produced by an autoregressive (AR) stochastic process of order $1 .^{7}$ Here the time evolution of the particle velocity is modeled as

$$
v(t+\Delta t)=a v(t)+\epsilon(t+\Delta t) .
$$

In the above AR model $a$ is a constant and $\epsilon(t)$ is white noise with amplitude $\sigma$. If Eq. (13) is multiplied by $v(0)$ and averaged over time one obtains a difference equation for $\psi(t)$, using that $\langle\epsilon(t+\Delta t) v(0)\rangle=0$,

$$
\psi(t+\Delta t)=a \psi(t) .
$$

One easily sees that Eq. (12) is the solution of the difference equation (14) for the initial condition $\psi(0)=1$. Setting $t=0$ in Eq. (14) one finds that

$$
a=\psi(\Delta t) .
$$

The noise amplitude $\sigma$ is obtained by multiplying Eq. (13) with $v(t+\Delta t)$, performing a time average, and assuming that $v(t)$ is a stationary time series, such that one can set $\left\langle v^{2}(t+\Delta t)\right\rangle=\left\langle v^{2}(t)\right\rangle \equiv\left\langle v^{2}\right\rangle$. The result is

$$
\sigma^{2}=\left\langle v^{2}\right\rangle\left(1-a^{2}\right) .
$$

It is clear that the amplitude of the error signal, which can also be viewed as prediction error of an optimal linear predictor, ${ }^{7} \widetilde{v}(t+\Delta t)=a v(t)$, becomes small if $a$ is close to unity, but not equal to unity. One can conclude that $\psi(\Delta t)$ should be representable by a Taylor expansion around $t=0$ which is truncated after the first term describing a deviation from $\psi(0)=1$. Using that $\dot{\psi}(0)=0$ and $\ddot{\psi}(0)=-\xi(0)$, one finds

$$
\psi(\Delta t) \approx 1-\frac{\Delta t^{2}}{2} \xi(0) .
$$

This approximation is valid if

$$
\Delta t \ll \frac{1}{\sqrt{\xi(0)}} .
$$

On the other hand, $a=\psi(\Delta t)$ should have the form given in Eq. (11). This yields the condition

$$
\gamma \Delta t=\frac{\Delta t^{2}}{2} \xi(0) .
$$

Using definition (8) of the friction constant it follows that

$$
\Delta t=2 \int_{0}^{\infty} d t \frac{\xi(t)}{\xi(0)} .
$$

The minimum step on the coarse grained time scale is thus given by twice the integral over the normalized memory function. From Eq. (20) it is obvious that $\Delta t$ does not change upon a scaling $\xi(t) \rightarrow \alpha \xi(t)$. In contrast, amplitude scaling of the memory function has an impact on condition (18). The latter would be increasingly better fulfilled with decreasing $\alpha$. This is exactly what we saw in the numerical study. ${ }^{5}$ 


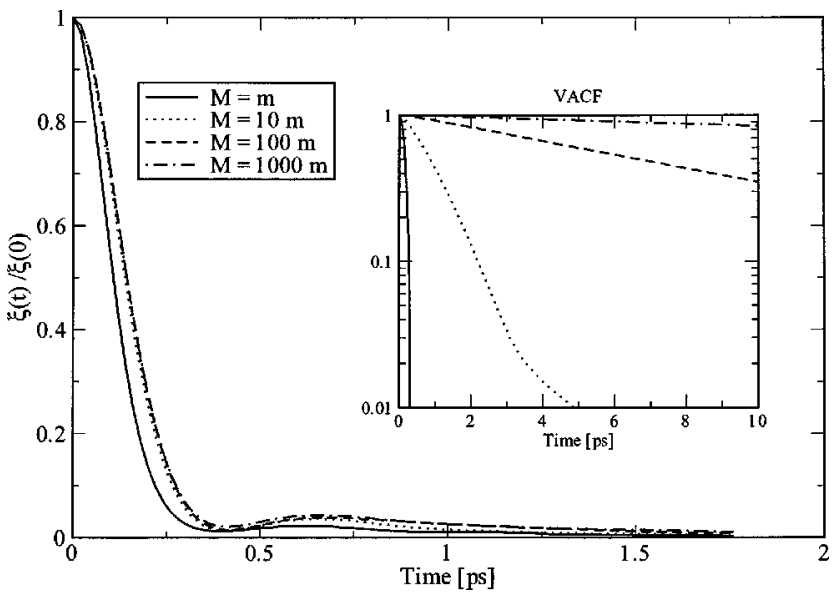

FIG. 1. The figure shows the normalized memory function $\xi(t) / \xi(0)$ for different masses of the tracer particle. Compared to the memory function of a normal argon atom, the scaling factors are $0.1003,0.0105,0.0013$ for tracer particles with $M / m=10, M / m=100$, and $M / m=1000$, respectively. More details are given in Ref. 5. The corresponding VACFs are shown in the inset, using a log-linear representation. We give here also the relative errors of $\psi(t)$ and $\xi(t) / \xi(0)$, which have been estimated from calculations for the three equivalent Cartesian components of the velocity of the tracer particle. For the above mass ratios (in increasing order) we obtain $\sigma_{\psi}=0.22,0.34$, $3.09,3.24 \%$ for the error of $\psi(t)$, and $\sigma_{\xi}=0.16,0.16,0.47,1.36 \%$ for the error of $\xi(t)$

Figure 1 shows the normalized memory functions and the corresponding VACFs of tracer particles which are immersed in a solvent consisting of 2047 molecules of liquid argon at a temperature of $90.0 \mathrm{~K}$. The tracer particles are modified argon molecules whose masses $M$ have been successively changed from $m$ to $1000 m$, where $m$ is the mass of one argon atom. Simulation details can be found in Ref. 5 . The figure shows that all memory functions have almost the same form. Only the one for $M=m$ has a slightly shorter range. The ratios of the memory function at time $t=0$ and the corresponding value for a normal argon atom are 0.1003, $0.0105,0.0013$ for $M / m=10, M / m=100$, and $M / m$ $=1000$, respectively. The corresponding theoretical values are $0.1005,0.0105$, and 0.0015 . The method we used to extract the memory function from the simulations has been described in detail in previous articles. ${ }^{1,2}$ The essential point is to represent the velocity of the tracer particle as an autoregressive stochastic process $v(t)=\sum_{n=1}^{P} a_{n}^{(P)} v(t-n \Delta t)$ $+\epsilon_{P}(t)$, where $P$ is the order of the AR process, $\Delta t$ is the sampling time step, the $\left\{a_{n}^{(P)}\right\}$ are constants, and $\epsilon_{P}(t)$ is white noise of zero mean. This step allows us to write down an analytical form for the (one-sided) $z$-transformed VACF $\Psi>(z)=\sum_{n=0}^{\infty} z^{-n} \psi(n \Delta t)=\sum_{k=0}^{P} \beta_{k} z_{k} /\left(z-z_{k}\right)$. Here the $\left\{z_{k}\right\}$ are the roots of the characteristic polynomial $p(z)=z^{P}$ $-\sum_{k=1}^{P} a_{k} z^{p-k}$ and the $\left\{\beta_{k}\right\}$ are constants which are computed from the roots $\left\{z_{k}\right\}$. One can now solve the $z$-transformed discrete version of the memory function equation (1) for the $z$-transformed memory function $\Xi_{>}(z)$ $=\left(1 / \Delta t^{2}\right)\left[z / \Psi_{>}(z)+1-z\right]$, and insert the analytical expression for $\Psi_{>}(z)$. The memory function in the time domain is then obtained by inverse $z$ transform which can be efficiently performed by polynomial division.

Table I shows for increasing mass ratios $\mathrm{M} / \mathrm{m}$ the characteristic Brownian dynamics time scale $\Delta t$ defined in Eq.
TABLE I. The table shows (columns from left to right) for different masses of the tracer particle the Brownian dynamics time step $\Delta t$ defined in Eq. (20), the value $1 / \sqrt{\xi(0)}$ appearing in condition (18), the friction constant $\gamma$ defined in Eq. (8), and the corresponding value obtained from a fit of $\psi(t)$ $\approx \exp \left(-\gamma_{\mathrm{fit}} t\right)$ to the VACF.

\begin{tabular}{rcccc}
\hline \hline$M / m$ & $\Delta t[\mathrm{ps}]$ & $1 / \sqrt{\xi(0)}[\mathrm{ps}]$ & $\gamma\left[\mathrm{ps}^{-1}\right]$ & $\gamma_{\mathrm{fit}}\left[\mathrm{ps}^{-1}\right]$ \\
\hline 1 & 0.2754 & 0.1378 & 7.3323 & 6.2288 \\
10 & 0.3486 & 0.4326 & 0.9314 & 0.9110 \\
100 & 0.3794 & 1.3374 & 0.1061 & 0.1024 \\
1000 & 0.3894 & 3.8483 & 0.0132 & 0.0131 \\
\hline \hline
\end{tabular}

(20) and the value $1 / \sqrt{\xi(0)}$ on the right-hand side of condition (18). In addition, the friction constant $\gamma$ and the corresponding value obtained from a least-squares fit of an exponential model to $\psi(t)$ are given. As all memory functions have a similar form, $\Delta t$ is similar in all cases. In contrast, the reference value $1 / \sqrt{\xi(0)}$ increases with increasing mass, such that the criterion (18) is fulfilled for very massive tracer particles. Correspondingly, the VACFs appear as straight lines in the log-linear representation shown in the inset of Fig. 1.

As already mentioned, the scaling behavior of $\xi(0)$ is exactly understood. Since $\ddot{\psi}(0)=-\xi(0)$ and $\ddot{\psi}(0)$ $=-\left\langle\dot{v}^{2}\right\rangle /\left\langle v^{2}\right\rangle$, it follows thus that $\xi(0)=\left\langle\delta F^{2}\right\rangle /\left(M k_{B} T\right)$, where $\left\langle\delta F^{2}\right\rangle$ is the mean square fluctuation of the force on the tracer particle. The mass $M$ has to be replaced by the reduced mass in case of a system with finite number of solvent molecules. ${ }^{5,6}$ In that light there exists a proof that the criterion (18) is systematically better fulfilled with increasing mass. It is worth noting that-similar to the simulation results-some analytical models also yield a global downscaling of the memory function with increasing mass of the tracer particle. ${ }^{8-10}$ In all cases the scaling behavior is a result of the respective forms for $\xi(t)$, which fulfil $\xi(0)$ $=\left\langle\delta F^{2}\right\rangle /\left(M k_{B} T\right)$ by construction. We note that this is one of the sum rules which can be derived for the memory function. For a concise and complete review we refer to the book by Boon and Yip. ${ }^{4}$

The above considerations explain why reducing the amplitude of the memory function leads formally to exponential VACFs. In this context we have presented formal criteria for Brownian motion which are derived from the memory function only. An essential point here is the introduction of a Brownian dynamics time step which is determined by the form of the memory function.

${ }^{1}$ G. Kneller and K. Hinsen, J. Chem. Phys. 115, 11097 (2001).

${ }^{2}$ T. Rog, K. Murzyn, K. Hinsen, and G. Kneller, J. Comput. Chem. 24, 657 (2003).

${ }^{3}$ R. Zwanzig, Statistical Mechanics of Irreversibility (Wiley-Interscience, New York, 1961), pp. 106-141, Lectures in Theoretical Physics.

${ }^{4}$ J. Boon and S. Yip, Molecular Hydrodynamics (McGraw-Hill, New York, 1980).

${ }^{5}$ G. Kneller, K. Hinsen, and G. Sutmann, J. Chem. Phys. 118, 5283 (2003).

${ }^{6}$ P. Español and I. Zuñiga, J. Chem. Phys. 98, 574 (1992).

${ }^{7}$ A. Papoulis, Probablity, Random Variables, and Stochastic Processes, 3rd Ed. (McGraw-Hill, New York, 1991).

${ }^{8}$ B. Berne, J. Boon, and S. Rice, J. Chem. Phys. 45, 1086 (1966).

${ }^{9}$ K. Singwi and M. Tosi, Phys. Rev. 157, 153 (1967).

${ }^{10}$ P. Damle and A. Tillu, Indian J. Pure Appl. Phys. 7, 539 (1969). 